Le LIDAR est un capteur de mesure à distance par laser.

Rapidement, avec le développement constant de nouvelles technologies laser plus performantes, miniaturisées

et fiables, plusieurs domaines d'application depuis le sol, et plus récemment depuis

l'espace, ont vu le jour.

Ils se fondent sur la mesure résolue en distance

de composants comme

les particules,

la vapeur d'eau ou l'ozone, ou de paramètres comme

le vent et la turbulence.

Les lidars sont actuellement

utilisés en routine pour

la surveillance du climat

et de la pollution ;

ils permettront dans un futur

proche d'augmenter

la productivité des champs

d'éoliennes, et d'améliorer

la prévision météorologique

et la sécurité sur les aéroports.

\title{
Les lidars atmosphériques
}

Jean-Pierre Cariou (jpcariou@leosphere.fr) et Laurent Sauvage

Leosphere, 14-16 rue Jean Rostand, Parc Club Université, 91400 Orsay

LIDAR est l'acronyme du terme LIght Detection And Ranging. C'est un instrument de sondage atmosphérique à distance, utilisant des ondes électromagnétiques optiques, de l'ultraviolet ( $\lambda=0,26 \mu \mathrm{m})$ à l'infrarouge $(\lambda=10 \mu \mathrm{m})$ [1].

Les premières mesures lidar concluantes ont été effectuées en 1962, afin de vérifier les possibles applications du laser à rubis développé en 1960 par Ted Maiman.

En France, le principe a acquis ses lettres de noblesse dans les années 70, avec son utilisation par Gérard Mégie pour le suivi de l'ozone stratosphérique et la surveillance du trou d'ozone, à l'Observatoire de Haute-Provence et dans des stations proches $\mathrm{du}$ cercle polaire arctique. Depuis, la technologie des lidars a suivi celle des lasers et a diversifié ses applications atmosphériques. Elle est désormais utilisée pour la caractérisation d'aérosols, de gaz, de champs de vent, ou la mesure de températures. Longtemps cantonnés aux laboratoires, les lidars sont, depuis peu, des outils industriels fiables et conviviaux, avec des applications en qualité de l'air, météorologie, sécurité aérienne et énergie éolienne.

\section{Principe des lidars}

Le lidar est un procédé de détection à distance, utilisant un laser. Celui-ci émet une impulsion brève de lumière (de 5 à 500 ns, correspondant respectivement à des résolutions spatiales de 1 à $100 \mathrm{~m}$ ), généralement monochromatique $(\Delta \lambda<1 \mathrm{~nm})$, qui se propage dans l'atmosphère. Le rayonnement émis va interagir avec les particules et les molécules de l'atmosphère, le long de la ligne de visée. Une partie de l'intensité diffusée est renvoyée dans la direction opposée à l'impulsion laser initiale, on dit qu'elle est rétrodiffusée. Ce signal rétrodiffusé s'étale dans le temps, proportionnellement à la distance des différentes couches traversées. Il est collecté à travers l'optique d'un télescope, et transformé par le système de détection optoélectronique en un signal électrique. Le lidar mesure l'intensité rétrodiffusée au cours du temps pour la caractérisation des aérosols et des gaz, et la fréquence du signal rétrodiffusé pour la mesure du décalage Raman et dans les lidars Doppler.

Il existe deux schémas de détection lidar : la détection incohérente (ou directe) et la détection cohérente (ou hétérodyne, utilisée pour la mesure de l'effet Doppler, cf. pp. 80-81). Pour les lidars à détection directe, le signal détecté est échantillonné à haute fréquence pour chaque impulsion, et cumulé dans un tableau. L'information temporelle, quant à elle, est multipliée par la vitesse de la lumière pour obtenir finalement le signal lidar en fonction de la distance. L'inversion de ce signal consiste à retrouver les profils des coefficients de rétrodiffusion et d'extinction de chaque couche atmosphérique. Comme le signal d'une couche dépend de l'absorption des couches inférieures, l'inversion utilise des algorithmes itératifs (méthode de Fernald-Klett) et des valeurs initiales déduites des modèles de profils moléculaires.

Deux types de diffusions sont rencontrées (voir encadré 1) :

- une diffusion élastique : le photon est diffusé sans changement de fréquence (au décalage Doppler près, induit par le mouvement des particules, voir encadré 2, p. 80). C'est le cas de la diffusion de Rayleigh et de la diffusion de Mie ;

- une diffusion inélastique, appelée aussi diffusion Raman. Celle-ci est à l'origine d'un décalage de la fréquence de l'onde diffusée par rapport à celle de l'onde incidente, et est propre à chaque molécule.

Pour une distance de mesure de l'ordre de $10 \mathrm{~km}$, le rapport entre le nombre de photons émis et le nombre reçu est de $10^{15}$ ! Il faut donc des lasers puissants (plusieurs dizaines de millijoules (mJ) par impulsion) et des photodétecteurs fonctionnant en comptage de photons pour atteindre des 


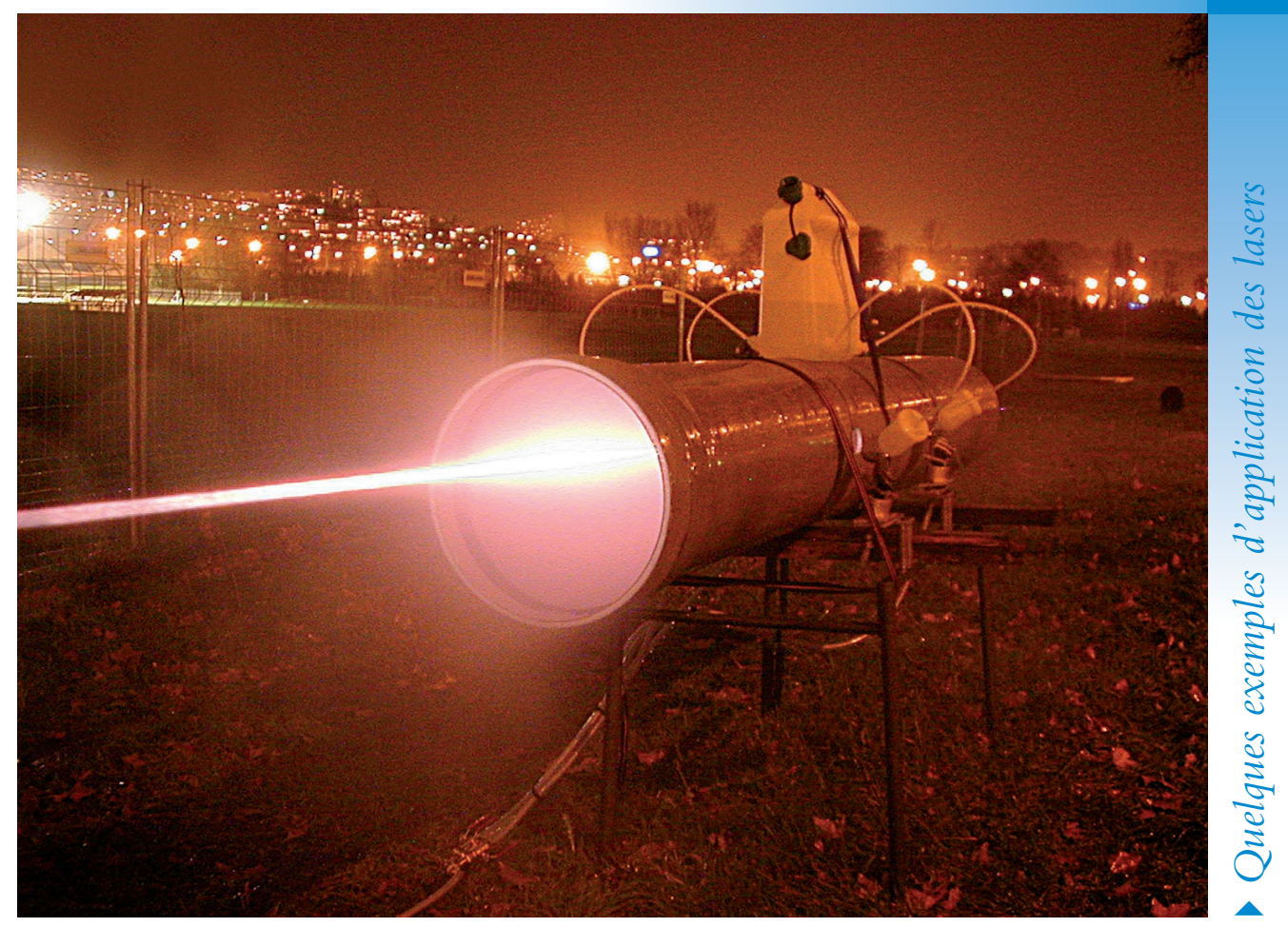

rapports signal sur bruit suffisants. La limite de sensibilité est toutefois souvent liée au flux de fond de ciel qui, malgré les filtres interférentiels de largeur inférieure au nanomètre, crée un signal parasite dont les fluctuations suivent une statistique de Poisson. La cadence de tirs laser est limitée à quelques kilohertz $(\mathrm{kHz})$, pour éviter d'émettre une nouvelle impulsion avant la réception de la précédente.

Par rapport au radar, le lidar offre une excellente directivité et résolution angulaire, bien adaptée à la cartographie 3D de structures (panaches, tourbillons, nuages). Par rapport aux caméras infrarouges, il offre, pour la détection de gaz, l'accès à une information en distance, et un fonctionnement indépendant des conditions de luminosité ou de température de la scène.

\section{Les applications du lidar}

Le lidar est surtout utilisé pour la recherche atmosphérique et la météorologie (mesure de la vitesse du vent par effet Doppler). Dans la suite de cet article, nous nous concentrerons sur ces deux applications.

Plus récemment, un certain nombre d'applications de topographie et de cartographie ont été mises au point, en utilisant le lidar en visée "vers le bas " à bord d'aéronefs ou de satellites.

En géologie et sismologie, un système utilisant un GPS et un lidar embarqués a donné naissance à un outil hautement fiable pour détecter les failles et les mouvements de terrain. La précision atteinte est remarquable : on peut ainsi mesurer l'élévation d'un terrain à travers les arbres comme, par exemple, pour la localisation de la faille de Seattle aux États-Unis.

Le lidar a également trouvé de nombreuses applications dans le domaine forestier : hauteurs de canopée (étage supérieur de la forêt), mesures de biomasse..., peuvent être obtenues à l'aide de systèmes embarqués.

Détection de simulants d’aérosols biologiques avec le Lidar Téramobile(t) : le faisceau laser arrive de la gauche, illumine le brouillard produit dans le tube et permet de l'analyser. O CNRS Photothèque / Université Jean Moulin Lyon 3 / GRATle Alexis. (1) Voir G. Méjean et al., Reflets de la physique n¹ (2006) 10-13.

\section{Les différents mécanismes de diffusion du rayonnement laser}

La diffusion élastique : régimes de Rayleigh et de Mie

La diffusion élastique se produit sans échange d'énergie entre les photons incidents et la particule rencontrée. Le photon est alors diffusé sans changement de fréquence, au décalage dû à l'effet Doppler près (cf. encadré 2, p. 80). On distingue la diffusion Rayleigh (lorsque la taille $r$ du diffuseur est largement inférieure à la longueur d'onde $\lambda$ ) et la diffusion de Mie $(r \sim \lambda)$. La section efficace de diffusion varie comme $(r / \lambda)^{4}$ pour le régime de Rayleigh, oscille fortement en régime de Mie, et varie en $(r / \lambda)^{2}$ pour les valeurs élevées de $r$ (régime géométrique). La diffusion de Mie diffère selon la polarisation si la particule est non sphérique, ce qui permet de discriminer sa nature (eau ou glace, sable ou pollution).

Variation de la section efficace de diffusion élastique d'un rayonnement laser par une particule sphérique, en fonction du rayon r de la particule $(\lambda=1550 \mathrm{~nm})$.

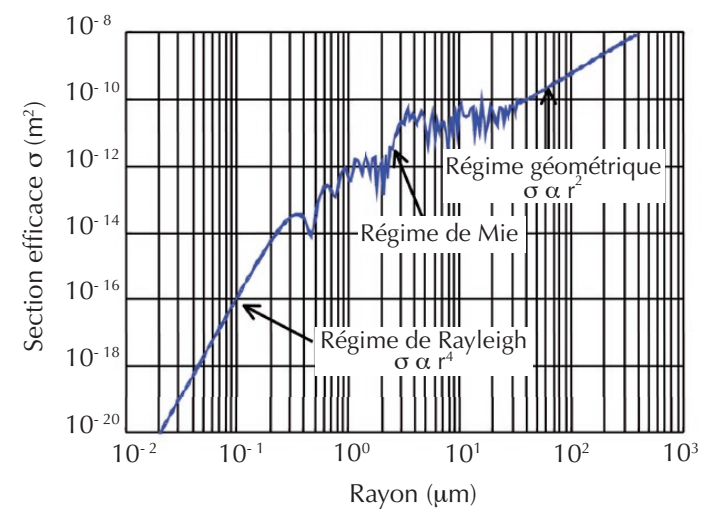

La diffusion inélastique (diffusion Raman)

La diffusion Raman consiste en la diffusion inélastique d'un photon par le milieu, qui retire (raie Stokes) ou ajoute (raie anti-Stokes) de l'énergie au photon et en modifie donc légèrement la fréquence. Cet échange provient de l'absorption ou de l'émission de quanta de vibration et rotation de la molécule, et est donc caractéristique du milieu. Le décalage en longueur d'onde ne dépend pas de la longueur d'onde d'excitation. La mesure consiste à sélectionner la lumière diffusée par un filtre interférentiel étroit $(\Delta \lambda \sim 1 \mathrm{~nm}$ ) autour du décalage propre au gaz à analyser $\left(\mathrm{N}_{2}, \mathrm{O}_{2}, \mathrm{H}_{2} \mathrm{O}\right.$...). Son inconvénient majeur est la faible section efficace de diffusion, qui nécessite un laser puissant et une détection très performante. 


\section{Le lidar pour les mesures atmosphériques}

Le changement climatique mondial est devenu l'une des principales menaces pour notre civilisation. En effet, en plus des émissions de particules naturelles, les activités anthropogéniques modernes rejettent dans l'atmosphère une énorme quantité de particules et de gaz à effet de serre. La concentration, l'âge, la taille, la forme, la nature et l'altitude de ces particules sont très variables. Ces paramètres impactent globalement et localement le bilan radiatif de plusieurs façons, par la diffusion ou l'absorption vers le bas et vers le haut des rayonnements émis par le soleil ou d'autres sources indirectes. Ainsi, il est largement reconnu (rapport du GIEC 2001) que les principales incertitudes sur la modélisation des changements climatiques sont liées à notre connaissance limitée des effets directs et indirects des aérosols (poussières minérales, combustibles fossiles, interaction aérosols-nuages).

Les nuages froids de haute altitude vont aussi limiter la modélisation du climat. Ils influencent grandement le bilan radiatif (par leur albédo ${ }^{(1)}$ ) et l'effet de serre, qui dépend de leur hauteur et de leurs propriétés optiques et microphysiques. Jusqu'à présent, la détection de ces nuages par des capteurs passifs était rendue difficile, en raison de leur altitude et de leur faible épaisseur optique.

Dans le domaine atmosphérique, le lidar est utilisé comme un instrument de détection à distance pour mesurer la densité de certains composants de la moyenne et de la haute atmosphère, tels que le potassium, le sodium, l'azote et l'oxygène moléculaires. On peut ainsi en déduire des températures.

Les lidars ont offert aux chercheurs une information nouvelle, en fournissant en continu des données sur la distribution verticale des aérosols et des couches nuageuses élevées. La surveillance résolue spatialement de la troposphère est primordiale pour les groupes de recherche impliqués dans la météorologie, l'astrophysique, les changements climatiques ou la qualité de l'air. La figure 1 montre un profil atmosphérique, mesuré en fonction du temps. On y distingue la couche limite atmosphérique ${ }^{(2)}$ et les différents types de nuages.

\section{Surveillance des aérosols}

Les aérosols troposphériques (jusqu'à $10 \mathrm{~km}$ ) proviennent de diverses sources, naturelles ou non (poussières, embruns, 544-545. www.nature.com/news/2010/100602 full/465544a.html 
cendres volcaniques, incendies de forêts, combustibles fossiles, combustion de moteurs, activités ou cultures agricoles, etc.). Ils sont très variables dans l'espace et le temps et ils peuvent avoir, directement ou indirectement, un impact (par l'interaction avec les nuages) sur le bilan radiatif. Leur localisation et caractérisation est aujourd'hui possible. Les lidars UV permettent la détection précise des différents aérosols, comme les poussières transportées du désert de Gobi vers l'est de la Chine, le Japon et même les ÉtatsUnis, les particules d'incendies de forêt transportées d'Amérique du Nord ou de Sibérie vers l'Europe... Plus la longueur d'onde est courte, plus le lidar est sensible aux petites particules. Ainsi, un lidar UV à $355 \mathrm{~nm}$ (laser YAG triplé en fréquence), tel que l'ALS450 développé par Leosphere (voir ci-après), peut mesurer aussi bien la concentration des aérosols de taille supérieure à $50 \mathrm{~nm}$ que celle des molécules d'azote ou de vapeur d'eau. Le signal de diffusion optique est traduit en extinction linéique ${ }^{(3)}$, par calibration sur la diffusion moléculaire ou par la connaissance de la transmission optique globale grâce à un photomètre. La transformation en concentration massique nécessite, en plus, une connaissance des propriétés micro- physiques des particules (granulométrie, indice complexe).

\section{Surveillance de la qualité de l'air}

La couche limite atmosphérique (jusqu'à 2 à $3 \mathrm{~km}$ ) contient la plupart des aérosols atmosphériques, en particulier les polluants émis par des sources locales ou éloignées (fig. 2). Il est indispensable pour l'analyse d'avoir la capacité d'enregistrer l'évolution rapide de la hauteur de cette couche d'inversion et des différentes strates résiduelles. On peut ainsi mieux représenter les réactions photochimiques et la dispersion des différents composants dans les modèles de prévisions météorologiques et de qualité de l'air environnant.

En outre, le contrôle de la concentration massique des particules au-dessus des zones polluées est actuellement un grave problème pour la plupart des preneurs de décisions de zones urbaines. La cartographie horizontale des taches de pollution (fig. 3) aide les autorités à améliorer l'efficacité de leur politique. Le lidar peut, par exemple, suivre en 3D la dispersion vers les habitations, de polluants provenant de sources locales (usines, routes). Enfin, comme les particules peuvent être utilisées comme traceurs des mouvements de l'atmosphère, le lidar peut y détecter des mouvements convectifs de cellules, des ondes de gravité( ${ }^{(4)}$, et d'autres effets dynamiques. Des mesures de faisabilité à courte distance, en intérieur dans un réseau souterrain, ont donné des résultats tout à fait probants. Les impulsions sont généralement courtes (10 ns), conduisant à des résolutions spatiales élevées $(1,5 \mathrm{~m})$.

\section{Surveillance des nuages}

Les effets locaux de refroidissement ou de réchauffement à chaque niveau de l'atmosphère dépendent de l'existence de nuages, de leur répartition, leur hauteur et leur morphologie, ainsi que de leurs propriétés optiques. Le comportement des nuages, la façon dont ils absorbent ou émettent les rayonnements visible et infrarouge, influencent fortement le bilan radiatif global de la Terre.

Le lidar atmosphérique ALS450 est issu de 15 ans de recherches (CNRS, CEA). C'est maintenant un système industriel fiable et validé. Toutefois, de nombreux développements sont toujours en cours, comme la mesure de la diffusion Raman, soit sur l'azote pour une calibration du système dans les hautes couches de l'atmosphère (absence d'aérosols), soit sur $\mathrm{H}_{2} \mathrm{O}$ pour connaître le taux de vapeur d'eau dans l'air.

\〉>

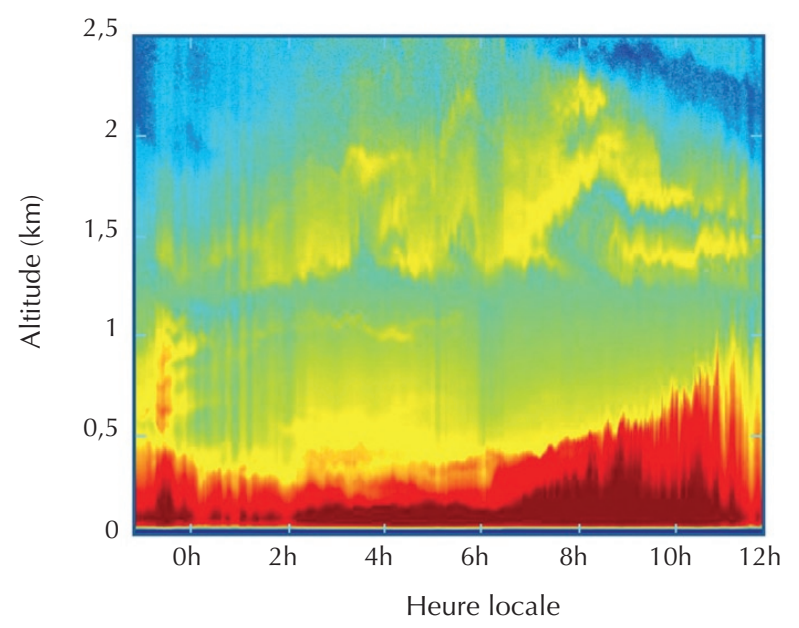

2. Stratification des aérosols dans la couche limite. (Code de couleur identique à celui de la figure 1).

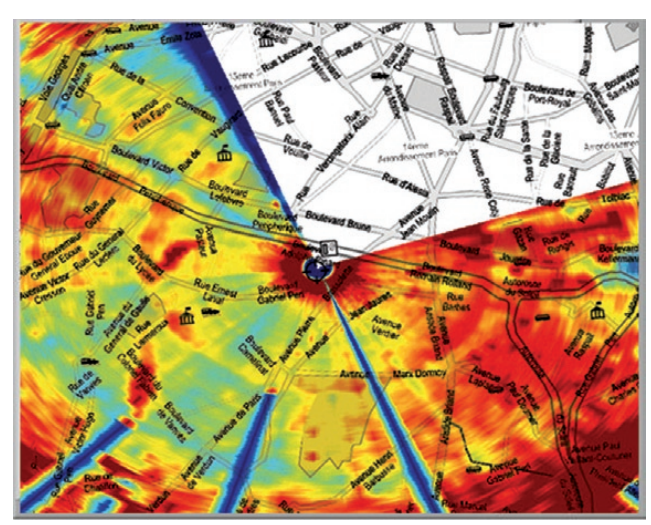

3. Carte de pollution mesurée avec un lidar aérosol, aux alentours du périphérique parisien. Les zones rouges indiquent de fortes concentrations. Les zones bleues proviennent de masques physiques sur le signal. 


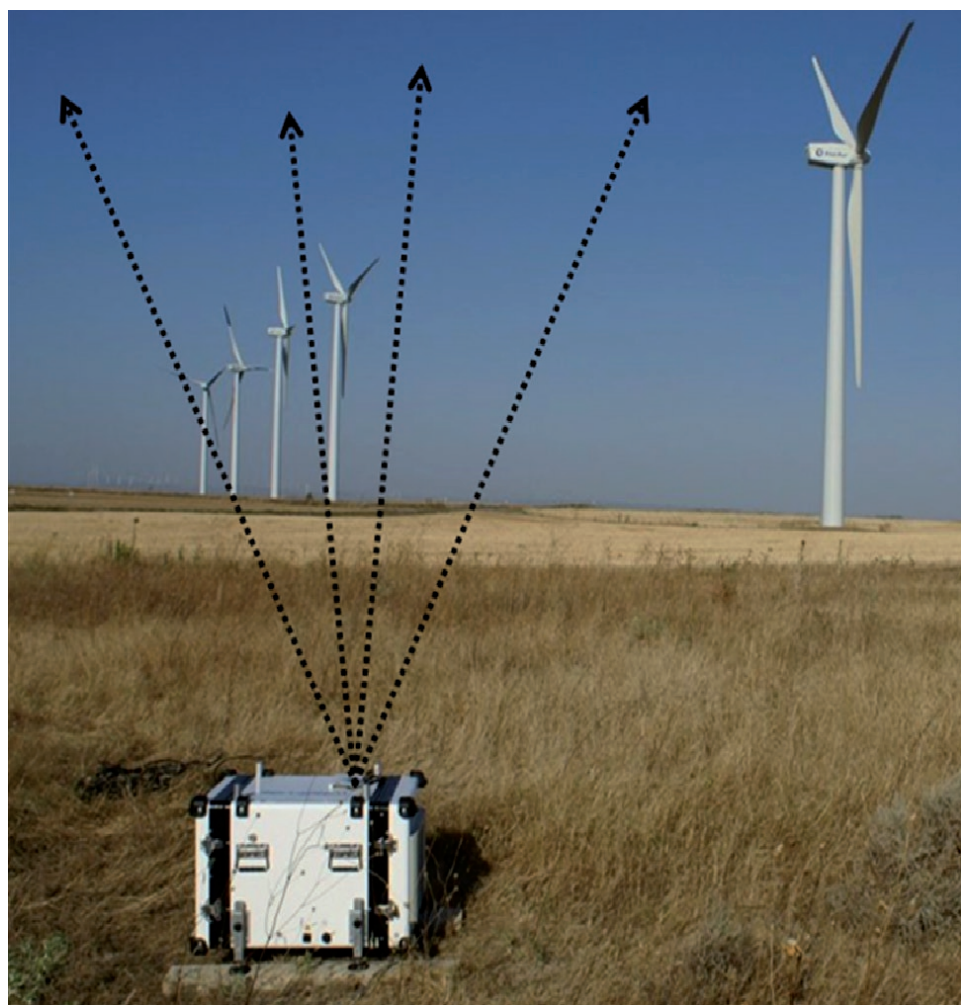

4. Lidar WINDCUBE sur site éolien. Les flèches indiquent les directions des quatre faisceaux laser, utilisés pour restituer les trois composantes du vecteur vent. Le WINDCUBE ${ }^{\mathrm{TM}}$ a été développé par Leosphere, avec une technologie ONERA pour ce besoin précis. II utilise un amplificateur laser à fibre optique à $1550 \mathrm{~nm}$, issu des technologies de télécommunications. Ce lidar a été testé et validé par des organismes indépendants, et a obtenu des performances inégalées en précision de vitesse $(0,1 \mathrm{~m} / \mathrm{s})$.

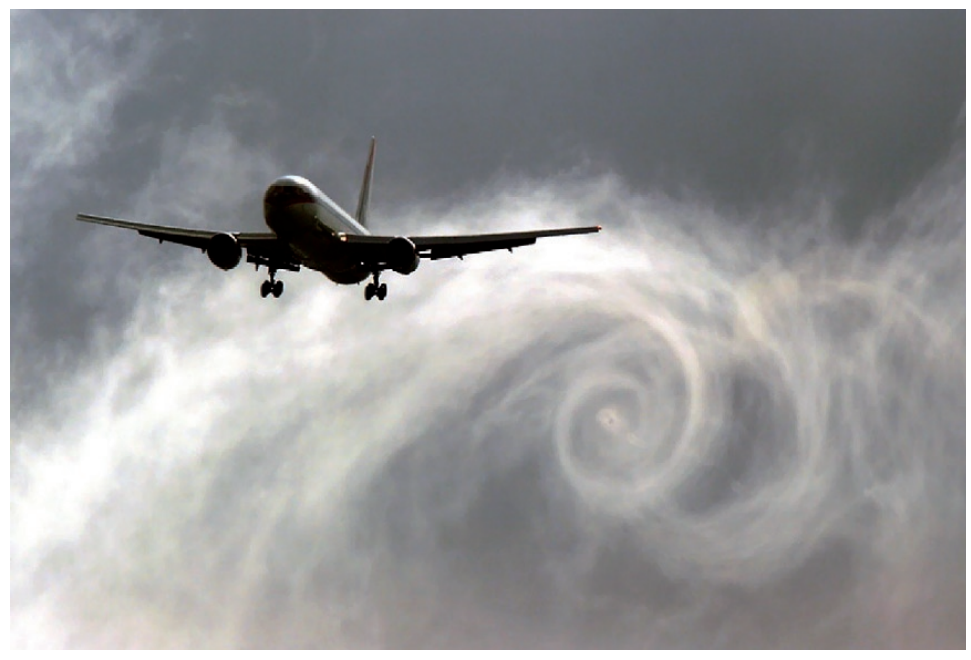

7. Tourbillon de sillage derrière un avion.

\section{$\gg\rangle$}

\section{Le lidar pour les mesures de vent}

Le lidar peut également être utilisé pour estimer la vitesse du vent, en mesurant le décalage fréquentiel Doppler (encadré 2) $\mathrm{du}$ laser, par diffusion sur les particules portées par le vent. Il remplace les mats de test pour les campagnes de mesures sur les champs d'éoliennes et procure un important gain de temps et d'argent.

La rentabilité d'un projet de parc éolien dépend de deux défis majeurs : la réduction de l'incertitude sur la production attendue, et la réduction du coût et des risques du projet. De plus, toutes les incertitudes concernant les prévisions des ressources en vent deviennent critiques.

Les concepteurs de turbine, les développeurs et les opérateurs ont besoin d'accéder à distance à de multiples données sur le vent : profil vertical, cartographie de la vitesse et de la direction, variations (cisaillement de vent et turbulences). Les mats de mesure actuels ont une hauteur de $60 \mathrm{~m}$, alors que le sommet des pales deséoliennes culmineà $200 \mathrm{~m}$. L'installation d'un mat nécessite un permis de construire ; certains sites complexes (ainsi évidemment que les sites en mer) ne peuvent pas en accueillir. L'installation facile des profileurs de vent lidar permet d'accélérer et de simplifier les procédures administratives. Au lieu d'extrapoler empiriquement les données du mat, le lidar mesure directement le vent à toutes les altitudes souhaitées. Sa petite taille permet également de le déplacer dans le parc éolien (fig. 4), à la différence des mats, fixes par nature. Une prochaine génération de « lidars vent " sera embarquée sur les turbines et permettra de mesurer en amont le vent incident, pour optimiser le rendement des éoliennes.

\section{Principe de fonctionnement}

Le lidar vent diffère du lidar aérosol par sa détection cohérente (ou hétérodyne, voir encadré 3). La lumière rétrodiffusée par les aérosols est collectée, et interfère avec

\section{L'effet Doppler}

L'effet Doppler-Fizeau est le décalage de fréquence d'une onde (électromagnétique ou acoustique) entre la mesure à l'émission et la mesure à la réception, lorsque la distance entre l'émetteur et le récepteur varie au cours du temps. Dans sa formulation galiléenne $\left(V_{r}<<c\right)$, la fréquence reçue $v_{\text {rec }}$ subit un décalage par rapport à la fréquence émise $v$, et vaut $: v_{\text {rec }}=\left(1-V_{r} / C\right) v$, avec $V_{r}$ vitesse radiale relative de déplacement. Dans le cas du lidar, la particule en mouvement voit l'onde laser avec un premier décalage de fréquence à sa réception, et rediffuse le rayonnement vers le lidar avec un second décalage identique. La fréquence Doppler associée est donc : $\mathrm{f}_{\mathrm{d}}=2 \mathrm{~V} / \lambda$, soit environ $1,3 \mathrm{MHz}$ par $\mathrm{m} / \mathrm{s}$, à $\lambda=1,5 \mu \mathrm{m}$. 
un oscillateur optique local pour créer un signal de battement (fig. 5). Le signal électronique, copie fidèle en amplitude et phase du signal optique, est numérisé et traité pour mesurer le décalage de fréquence Doppler induit par les mouvements des aérosols.

On peut ainsi effectuer une mesure absolue de la vitesse radiale du vent, selon la ligne de visée du lidar. La résolution en altitude provient de la mesure du temps de propagation des impulsions. La résolution spatiale est constante sur toute la portée, grâce à l'utilisation d'une source impulsionnelle. Outre la position de la fréquence Doppler, l'élargissement spectral donne accès à la turbulence locale, et l'intensité du signal de retour à la concentration relative des aérosols.

\section{Récupération du vecteur vent $3 D$}

Le lidar Doppler mesure uniquement la composante du vent projetée sur l'axe de visée (vitesse radiale).

Pour restituer les trois composantes du vecteur vent, quatre lignes de vue sont successivement analysées et les mesures sont combinées (fig. 6). Pour des impulsions de 200 ns, le champ de vent récupéré correspond à une moyenne sur une couche atmosphérique d'environ $20 \mathrm{~m}$ d'épaisseur, centrée sur au moins dix altitudes, de 40 à $220 \mathrm{~m}$ (application de prospection d'énergie éolienne). Chaque axe de visée est sondé en une seconde, pendant laquelle 20000 impulsions sont émises. Le traitement d'analyse spectrale est effectué en temps réel par un processeur dédié.

En augmentant la puissance du laser et la taille du télescope, il est possible de mesurer le vent à des altitudes plus importantes, jusqu'à $10 \mathrm{~km}$. Dans les applications météorologiques, on étudie les phénomènes de convection dans la couche limite. Cette avancée intéresse également les responsables de sécurité aérienne pour une détection en temps réel, sur les aéroports, des turbulences naturelles ou créées par les avions (rafales, tourbillons de sillage (fig. 7), cisaillements de vent, etc.) [2].

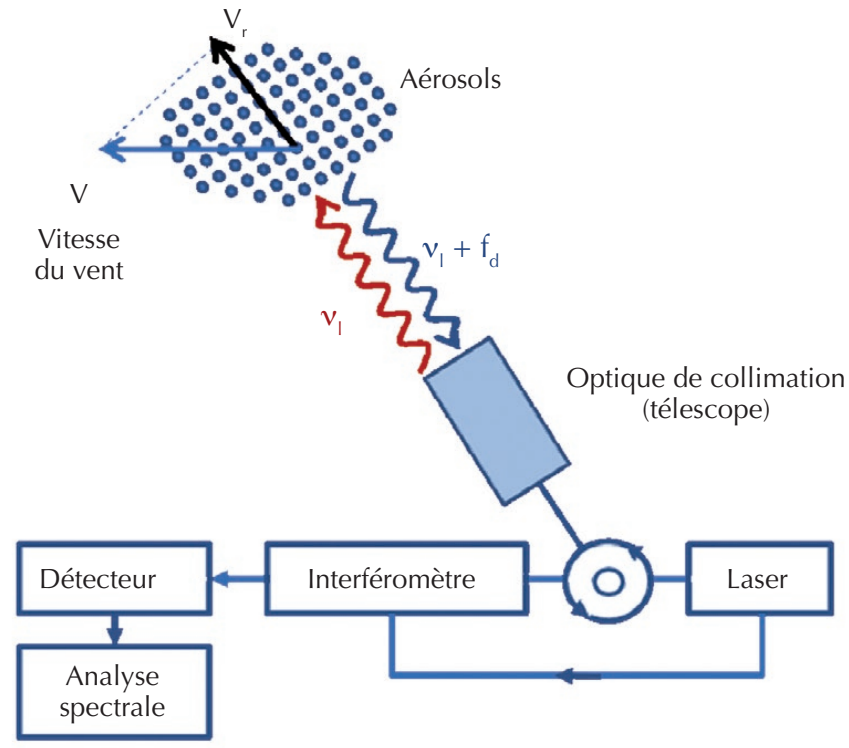

5. Principe du lidar Doppler hétérodyne.

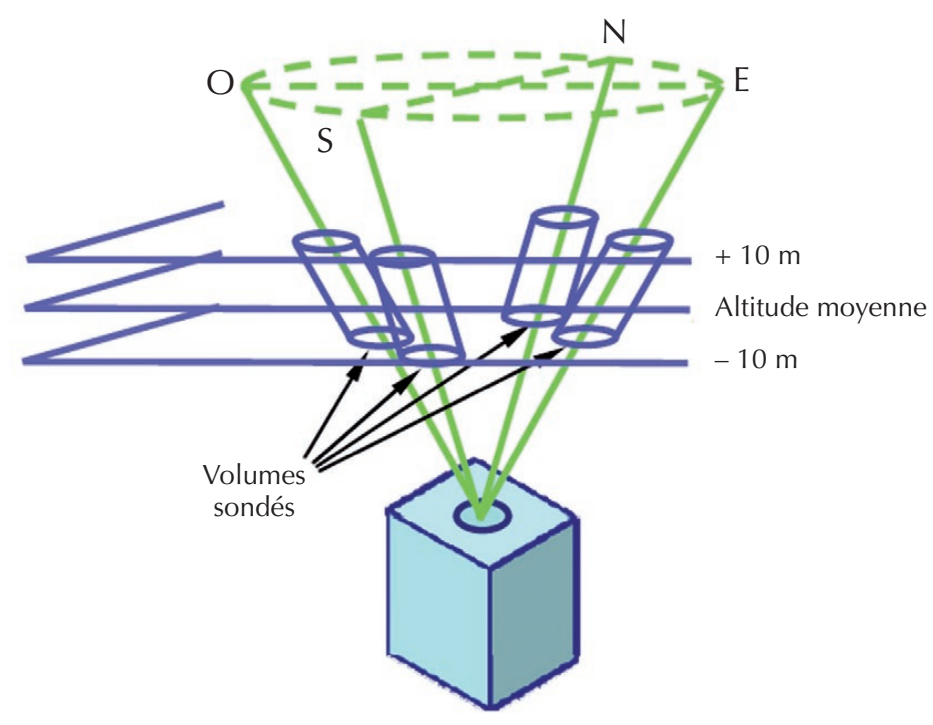

6. Reconstruction du vecteur vent avec le lidar Doppler WINDCUBE.

\section{Détection cohérente (ou hétérodyne)}

L'hétérodynage est une technique de transposition dans le spectre des radiofréquences d'un signal optique, qui conserve ses propriétés d'amplitude et de phase. Elle consiste à superposer sur un détecteur l'onde signal à analyser avec une fraction d'une onde de référence, classiquement issue du laser d'émission (fig. 5, droite). La fréquence différence est assimilable à une fréquence de battement, comme celle audible entre deux instruments légèrement désaccordés. Ce principe permet de mesurer les très petits décalages de fréquence comme ceux obtenus par décalage Doppler sur des particules se déplaçant à quelques $\mathrm{m} / \mathrm{s}$ (encadré 2). Une grande exigence est nécessaire, aussi bien quant à la qualité spectrale de la source (meilleure que l'inverse de la durée de l'impulsion) qu'en ce qui concerne la qualité spatiale des fronts d'onde proches des limites de diffraction. Un avantage décisif de la détection cohérente est d'être limitée par le bruit de photon du signal, même avec des détecteurs non parfaits. 


\section{\>> \\ La mesure des particules volcaniques}

Lors de l'épisode du passage du nuage volcanique islandais Eyjafjallajokull, en avril 2010, les lidars aérosol et Doppler ont été mis à contribution, les premiers mesure de la concentration des couches de cendres, les seconds pour la mesure de la vitesse de déplacement et la prévision de la trajectoire du nuage [3]. pour la détection, l'identification et la
La figure 8 montre le passage du nuage à la verticale de Paris, du 17 au 19 avril. Afin de distinguer le nuage de cendres d'autres nuages, une mesure en polarisation croisée avec la polarisation linéaire d'émission est effectuée. Le rapport de dépolarisation mesure la non-sphéricité des aérosols et aide à leur identification. Le nuage, étalé sur $1 \mathrm{~km}$ d'altitude, descend progressivement au-dessus de la station de mesure (ellipse orange). La partie basse du nuage (ellipse rouge) se fond et se dilue dans la couche limite atmosphérique (zone blanche) à partir du 19 avril.

La figure 9 illustre la capacité du lidar Doppler à mesurer le vent à haute altitude. Les barbules superposées à la carte de densité relative du nuage volcanique, indiquent la force et la direction du vent horizontal aux différents points, permettant de prévoir la trajectoire future du nuage. Le 17 avril 2010, le vent vient du nord-est le matin, et tourne au nord-ouest le soir.

\section{Conclusion}

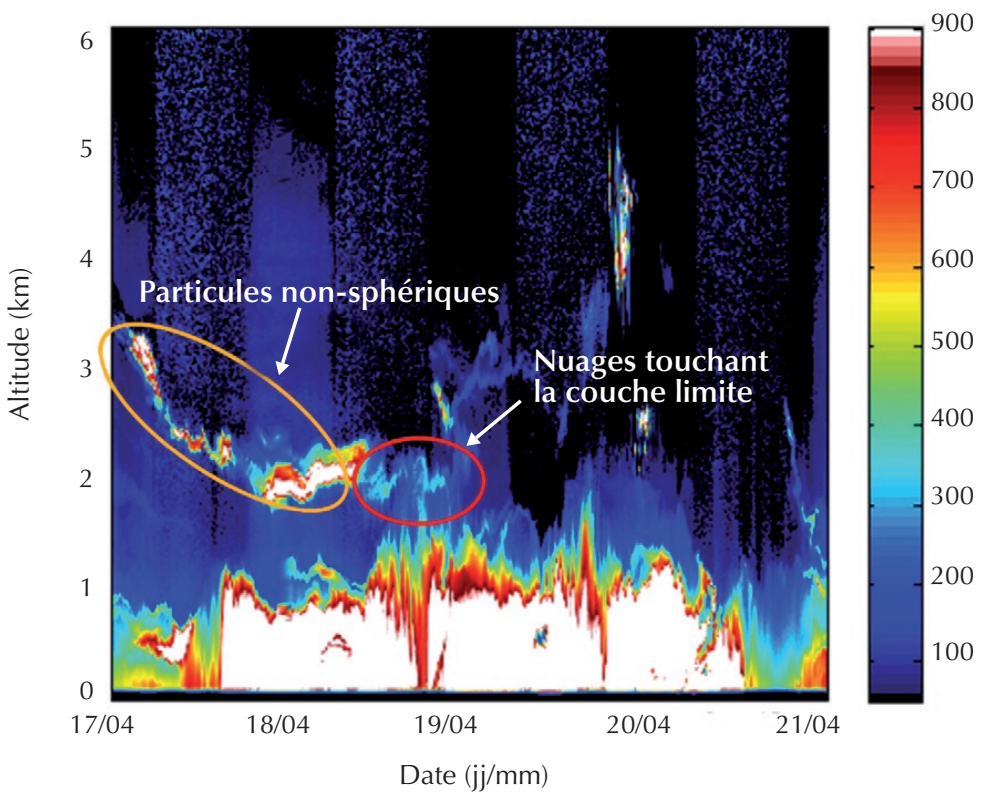

8. Observation de l'arrivée du nuage volcanique islandais au-dessus de Paris, en avril 2010, avec un lidar aérosol ALS450 (longueur d'onde $355 \mathrm{~nm}$, résolution spatiale $15 \mathrm{~m}$, résolution temporelle $30 \mathrm{~s}$ ).

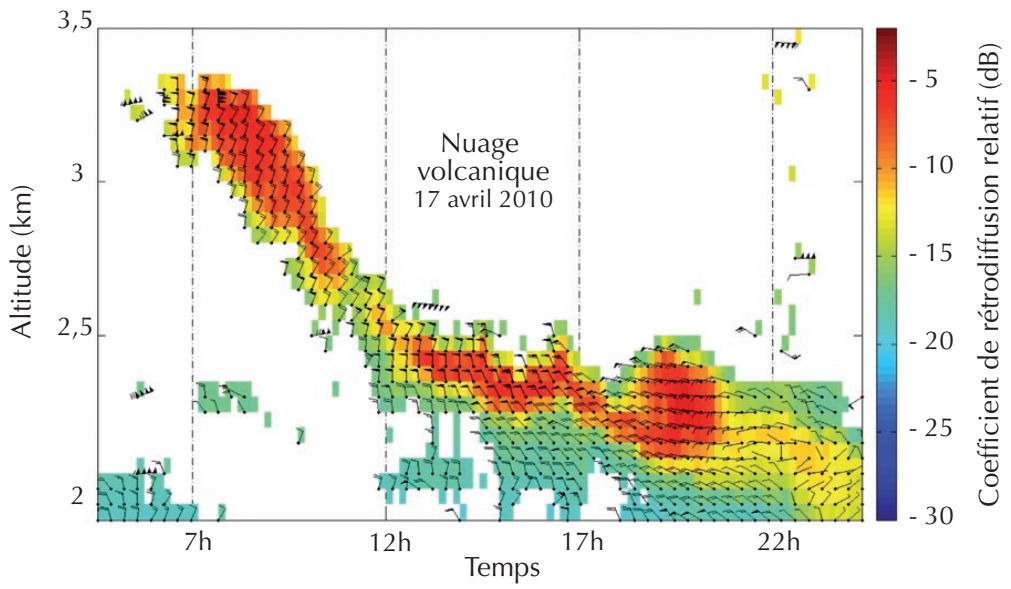

9. Champ de vent dans le nuage volcanique islandais mesuré en fonction du temps et de l'altitude, superposé à la carte de densité relative du nuage. Mesures effectuées avec un lidar Doppler WINDCUBE 200, le 17 avril 2010, dans la zone correspondant à la partie gauche de la figure 8 .
Les lidars ont pleinement bénéficié des avancées des lasers au cours du demi-siècle passé. C'est cependant dans le sondage atmosphérique que le lidar a le plus évolué, chaque progrès technique, chaque nouvelle longueur d'onde trouvant son application dans la mesure des gaz, des particules ou du vent. Grâce à la montée en puissance des diodes laser, des barreaux de verres dopés et des fibres optiques amplificatrices, des systèmes lidar compacts et fiables ont pu être récemment développés industriellement pour servir les marchés de la qualité de l'air, de la météorologie, de l'énergie éolienne et bientôt de la sécurité aérienne. Des lidars spatiaux ont été ou vont être lancés (Calypso 2008, Aladin-Aeolus 2012) pour fournir une cartographie globale des aérosols atmosphériques et du vent sur la planète. Orienté sur des problématiques sociétales actuelles (climat, qualité de l'air, énergies renouvelables), le domaine reste également actif au niveau de la recherche académique, où de nouveaux principes et nouveaux composants sont en permanence évalués et améliorés.

(1) L'albédo est une grandeur sans dimension, rapport de l'énergie solaire réfléchie par une surface à l'énergie solaire incidente.

(2) La couche limite atmosphérique (CLA) est la zone de l'atmosphère entre la surface (terre ou mer) où la friction ralentit le déplacement de l'air, et l'atmosphère libre, où cette friction devient négligeable. Du fait de l'inversion du gradient de température à son sommet, elle concentre la majorité des particules créées au niveau du sol, et donc les polluants.

(3) Le coefficient d'extinction linéique est la fraction, par unité de longueur, du flux prélevé (par absorption ou diffusion) par l'atmosphère dans la direction initiale de propagation.

(4) Dans ce contexte, on appelle onde de gravité une onde de pression de l'air, à ne pas confondre avec une onde gravitationnelle. 